\title{
Heart-rate reactivity to reminder treatment predicts test performance in rats given ECS following training
}

\author{
TIMOTHY K. WITTMAN and TERRY L. DeVIETTI \\ Central Washington University, Ellensburg, Washington 98926
}

\begin{abstract}
Two experiments compared the capacity of reminder treatments to modify retention performance in rats either strongly trained and given ECS or weakly trained and given no ECS. Heart rate was monitored during the reminder sessions. In Experiment 1, the factorial combinations of stimulation of the mesencephalic reticular formation (MRF) (present, absent) followed by the presentation of the cue originally paired with footshock in training, a tone (present, absent), were evaluated as reminder stimuli. Results indicated that both groups showed decreased heart rate to the presentation of the tone in the reminder session. However, in the retention test, ECS animals given tone reminder showed increased retention, whereas weakly trained animals showed decreased retention. Experiment 2 used a shorter interval to onset of the tone in the reminder session. Heart rate to the reminder tone again decreased in weakly trained animals, but no change was obtained in ECS animals. No change in test performance as a result of reminder was noted in either group. These results support the notion that ECS following training influences memory retrieval systems and suggest that heartrate reactivity may be used to predict the behavioral effects of reminder treatment in ECS animals.
\end{abstract}

Reminder treatments interpolated between training and a retention test quite consistently increase retention performance in animals given an amnesic treatment following training. In most instances, this reminder effect is not obtained in, for example, animals trained but not given amnesic treatment or animals not trained (e.g., DeVietti \& Hopfer, 1974). However, controversy exists as to the meaning of this effect. Some regard the reminder literature as suggesting that amnesic treatments affect memory retrieval processes (e.g., DeVietti \& Hopfer, 1974; Lewis, 1969, 1976; Miller \& Springer, 1973, 1974). Others (e.g., Cherkin, 1972; Gold, Haycock, Macri, \& McGaugh, 1973; Gold \& King, 1974; Kesner \& Conner, 1974) point out that such data can be assimilated into the idea that amnesic treatments disrupt memory storage by assuming that a remnant of memory survives the amnesic treatment and that the reminder treatment acts as a weak training trial. It follows that these two weak memories, the training experience and the reminder, would generalize and summate. This notion is known as the generalization hypothesis, and data have been reported which appear to support this position. For example, several studies have shown that a reminder treatment increases retention performance both in ECS-treated animals and in animals that received weak training but no ECS (Gold et al., 1973; Haycock, Gold,

This research was supported in part by National Science Foundation Grant BMS73-07038-A01. Requests for reprints should be sent to Terry L. DeVietti, Department of Psychology, Central Washington University, Ellensburg, Washington 98926.
Macri, \& McGaugh, 1973). The parallel effects of reminder are consistent with the generalization hypothesis, but, as pointed out earlier (DeVietti \& Haynes, 1975), this argument is by analogy, and parallel improvements in retention performance could result from different mechanisms operating in the two groups instead of from a common mechanism. Our laboratory has reported two experiments in which reminder treatments increased retention performance in ECS-treated animals but had little, if any, effect on the performance of weakly trained animals (DeVietti \& Haynes, 1975; DeVietti \& Kirkpatrick, 1978). In both of these experiments, however, the reminder treatment conditions were arranged in such a way that one would be hard pressed to argue that these reminder events did not constitute a learning trial, inasmuch as the reminder treatment consisted of the presentation of either prior training cues followed by footshock $1 \mathrm{~h}$ later (DeVietti \& Haynes, 1975) or training cues followed immediately by stimulation of the mesencephalic reticular formation (MRF) (DeVietti \& Kirkpatrick, 1978). If these treatments resulted in new learning, the meaning of the differential results obtained between animals that received ECS and weakly trained animals is not as clear as one might like. The problem, therefore, is to define a reminder treatment that does not result in new learning that is related to the previous training experience. While this condition may be impossible to obtain, it may be possible to succeed in arranging the reminder stimuli in such a way that defines a backward rather 
than a forward conditioning trial. Such an approach was used by Quartermain, McEwen, and Azmitia (1972). They trained mice in a passive avoidance task and used cycloheximide as the amnesic agent. A reminder footshock was given $1 \mathrm{~h}$ before the first retention test, and this condition of footshock and testing defined the reminder treatment. Mice trained and given cycloheximide showed increased retention performance in a second test given $4 \mathrm{~h}$ after the reminder treatment. In the present experiment, we used this general approach by preceding the presentation of the tone used in original training by stimulation of the MRF as reminder and testing the effects of this procedure in ECS-treated and weakly trained animals. Previous research (DeVietti \& Kirkpatrick, 1978) had demonstrated that the presentation of the tone followed by MRF stimulation was an effective reminder treatment in ECS animals. In addition, heart rate was recorded during the reminder session in order to gain some insight into the degree of autonomic activation resulting from this procedure.

\section{EXPERIMENT 1}

\section{Method}

Animals and Apparatus. The animals were male Long-Evans rats from the Central Washington University Psychology Department colony, 90-120 days of age at the beginning of the experiment. The basic apparatus has been described in detail elsewhere (DeVietti \& Larson, 1971). Briefly, two identical chambers were used for fear conditioning, reminder treatment, in some instances, and testing. They measured $20 \times 30 \mathrm{~cm}$ and were $30 \mathrm{~cm}$ high. The sides and top were Plexiglas and the back and front were aluminum painted flat gray. The grid floor was .64-cm-diam stainless steel rods spaced $1.9 \mathrm{~cm}$ apart. A small hole just off center in the front wall allowed the insertion of a drinking tube; the orifice was located approximately $5 \mathrm{~cm}$ inside the chamber and $5 \mathrm{~cm}$ above the grid floor. A drinkometer circuit was formed between the water in the drinking tube and the grid floor. A $10-\mathrm{cm}$ loudspeaker for delivery of the conditioned stimulus (a 15-sec, 1,200-Hz, 90-dB tone from a Heathkit tone generator) was positioned at floor level behind the rear wall of each chamber. Both chambers were individually enclosed in lighted, fan-ventilated, sound-attenuation boxes. A chamber used to administer noncontingent treatments had walls constructed entirely of Plexiglas $(21 \times 56 \times 21 \mathrm{~cm}$ high) with a grid floor of the same size and spacing as those in the training-testing chambers. Scrambled footshock, delivered through the grid floor of the conditioning chamber was either "strong" $(1.6 \mathrm{~mA}, 3 \mathrm{sec}, 60 \mathrm{~Hz}, 330 \mathrm{~V} \mathrm{rms})$ or "weak" (1.0 mA, $1 \mathrm{sec}$, $60 \mathrm{~Hz}, 206 \mathrm{~V} \mathrm{rms})$. Strong footshock was also administered in the noncontingent treatment chamber. An ECS apparatus (Hayes, 1948) was set to deliver a $92-\mathrm{mA}$ shock $(200 \mathrm{msec}, 60 \mathrm{~Hz}, 1,840 \mathrm{~V} \mathrm{rms})$ to the ears of the rat through wire clips fashioned to interlock with baby clothes snaps (Prims No. 0) previously fitted to each ear. A BERL constant current stimulator in series with a micro current amplifier was set to deliver a 60 -sec train, $6 \mathrm{sec}$ on $/ 3 \mathrm{sec}$ off, of a $15-\mu \mathrm{A}, 300-\mathrm{Hz}, 1-\mathrm{msec}$ biphasic rectangular pulse with a 1 -msec interpulse interval. Heart-rate recordings were obtained with a small, molded Plexiglas saddle fitted to the rat in "packanimal" fashion. This saddle had alligator clips that were attached to thoracic electrodes and, through leads, to a Gilson polygraph recorder.

Surgery and Histology. The animals were anesthetized with pentobarbital $(40 \mathrm{mg} / \mathrm{kg}$ ) and implanted, unilaterally, with electrodes aimed at the MRF. Following system " $B$ " of Pellegrino and Cushman (1967), the implantation coordinates were $4.0 \mathrm{~mm}$ posterior to bregma, $2.00 \mathrm{~mm}$ lateral to midline, and $7.00 \mathrm{~mm}$ below the surface of the cortex. Bipolar electrodes were obtained from Plastic Products and were constructed of .25-mm stainless steel wire. Stainless steel safety pins (No. 1) were fitted subcutaneously along both sides of the thorax and served as electrodes for the recording of heart rate. Following surgery, the animals received $.08 \mathrm{cc}$ penicillin-G combiotic. Upon completion of the behavioral portion of the experiment, the animals were killed and perfused with saline, followed by $11 \%$ Formalin solution, and the electrode placements were confirmed following the method of Thomson, Beeman, Conger, Reeder, Steinhilb, Wittman, and DeVietti (1978).

Procedure. Following surgery, the animals were individually housed and maintained on ad-lib food and water. Ten to 14 days later, the animals were handled for $5 \mathrm{~min}$. Then they were fitted with the heart-rate recording saddle and allowed to move about a table top for $5 \mathrm{~min}$, once a day for 3 days. Following this adaption period, the animals were fitted with ECS electrodes by placement of a baby-clothes snap (Prims No. 0) in each ear. During the next 4 days, the animals were given experience with the various aspects of the experimental situation according to the following schedule. On the first day, each rat was placed into one of the two training-testing chambers and left there for $10 \mathrm{~min}$. During this interval, water was removed from the home cage. The following day, each rat was taken to the chamber, its nose was gently touched to the drinking tube now present, and it was then released near the rear of the chamber. After the subject had completed a total of $55 \mathrm{sec}$ of contact with the drinking tube, it was returned to its home cage, where water was again available. On the third day, the subject was fitted with the saddle, the MRF electrode cable, and the ECS leads; it was then placed in the chamber for $1 \mathrm{~min}$, and the home cage water was removed. The next day, the subject was again allowed to complete $55 \mathrm{sec}$ of contact with the drinking tube. The drinking tube was present in the chambers only during the two drink training days described above and on the test day described below.

On Day 5, the subjects were assigned randomly to one of four treatment conditions. Subjects in one group (TSFS/ECS) had the ECS leads attached and were placed separately into one of the two training-testing chambers. One minute later, the 15 -sec tone was presented and a strong footshock, delivered through the grid floor, was administered during the last $3 \mathrm{sec}$ of the tone. ECS followed $.5 \mathrm{sec}$ after offset of the tone and footshock. Subjects in two other groups were treated identically, except that no ECS was delivered. One group (TSFS) received strong footshock paired with the tone, and the other group (TWFS) was given weak footshock paired with the tone. The weak footshock parameters previously had been shown to yield test latencies similar to those of the TSFS/ECS subjects (DeVietti \& Haynes, 1975). Subjects in the fourth group (NCSFS/ECS) were placed in the noncontingent treatment chamber and received strong footshock and ECS. This group served as control for any "systemic" effects of footshock and ECS.

Four days following training or noncontingent footshock, animals in each of the four major groups were given reminder treatment. The animals were fitted with the heart-rate recording saddle and the MRF electrode cable. Reminder treatment consisted of the factorial combinations of MRF stimulation (present, absent) and presentation of the tone used in training (present, absent). Animals assigned to the tone condition were placed in the original training apparatus for reminder and animals assigned to the notone conditions were placed in a cardboard box in a different area of the laboratory. The reminder session lasted $180 \mathrm{sec}$ with stimulation or no stimulation of the MRF administered during the second $60 \mathrm{sec}$ of the session. Fifteen seconds of tone or no tone was presented $15 \mathrm{sec}$ after the MRF stimulation or no-stimulation offset. Heart rate was recorded during the first $60 \mathrm{sec}$ of the session the $15 \mathrm{sec}$ following MRF stimulation or no stimulation, and the $15 \mathrm{sec}$ of the presentation of the tone or no tone, and continued for the remaining $30 \mathrm{sec}$ of the session. 
All animals were tested after $24 \mathrm{~h}$ of water deprivation the day following reminder treatment. The testing procedure was started by gently touching the animal's nose to the drinking tube in the chamber and releasing the rat near the rear of the chamber. After the animal had completed $50 \mathrm{sec}$ of tube contact, the tone was automatically presented and remained on until the animal had completed an additional $5 \mathrm{sec}$ of tube contact. The time (in seconds) for the animal to complete $5 \mathrm{sec}$ of tube contact in the presence of the tone was automatically recorded and served as data for analysis. Animals not drinking within $600 \mathrm{sec}$ after tone onset were removed from the apparatus and assigned a score of 600 .

\section{Results}

The histology revealed that the electrode tips found to be located in the MRF clustered in an area bounded dorsally by the superior colliculus, laterally by the medial geniculate, ventrally by the medial lemniscus, and medially by the central gray.

Analyses of both the behavioral test data and the heart-rate reactivity data obtained during the reminder session are presented. Differences in sample size among groups reflects the fact that not all animals had proper electrode placement as well as the loss of animals because of equipment failure or ECS-induced paraplegia. Also, differences in sample size in various groups between heart-rate and behavioral analysis resulted because not all heart-rate records were interpretable.

Analysis of drink latencies. Latencies to drink in the presence of the test tone were more variable than any we have collected previously using this task. The increase in test score variability may have resulted from the procedure necessary to record heart rate, that is the insertion of safety pins under the dermis along the thorax with wearing of the saddle, although the animals were not encumbered by the saddle in the test. The drink latencies in the presence of the test tone were evaluated with a 2 by 2 by 4 unweighted means analysis of variance (Kirk, 1968), with stimulation of the MRF (present or absent) in the reminder session, tone (present or absent) in the reminder session, and groups differentiated in training serving as main effects. The data are shown in Table 1. The analysis revealed an effect of groups $[F(3,82)=13.17, p<.01]$ and an interaction between tone (present, absent) and groups $[F(3,82)=2.69, p=.05]$. No other source of variance approached statistical reliability. The analysis of the simple main effects of the Tone by Groups interaction, pooled across the MRF stimulation variable, indicated that TWFS animals given the tone in reminder showed decreased drink latencies compared with similarly trained animals not given the tone in the reminder session $(p<.05)$. The same comparisons in both the TSFS and NCSFS/ECS animals, while in the same direction, yielded values which did not achieve statistical reliability.

The drink-latency performance of the TSFS/ECS animals stood in sharp contrast with the pattern shown by the other three groups. TSFS/ECS animals given the tone in the reminder session showed increased drink latencies in the test compared with similarly trained animals not given the tone in reminder $(p<$ .05 , one-tailed).

Also, as part of the analysis of the interaction, differences were obtained among the four groups given tone reminder as well as among the four groups not given tone reminder. Comparisons between groups not given tone reminder, using the Newman-Keuls statistical test, indicated longer drink latencies in the TSFS animals than in both the NCSFS/ECS and TSFS/ECS animals ( $p s<.05$ ), but no difference was obtained between this group and the TWFS animals. TWFS animals showed longer drink latencies than NCSFS/ECS animals $(p<.05)$. No other comparisons were significant.

The results of the comparisons among the four groups given the tone in the reminder session revealed a different pattern of results. Here, TSFS animals had longer drink latencies than either NCSFS/ECS or TWFS animals (ps $<.05$ ), but did not differ from TSFS/ECS animals. Also, TSFS/ECS animals had longer drink latencies than either NCSFS/ECS or TWFS animals ( $\mathrm{ps}<.05$ ).

Analysis of heart-rate reactivity. This experiment was designed, in part, to evaluate heart-rate reactivity to the various components of the reminder session, that is, MRF stimulation and presentation of the tone. Accordingly, we recorded heart rate at various times during the reminder session: $15 \mathrm{sec}$ following MRF stimulation or no stimulation, during tone or no-tone presentation, as well as before and following these intervals. Heart rate was counted manually from the strip charts by one of the experimenters after the con-

Table 1

Drink Latencies in the Presence of the Test Tone in Experiment 1

\begin{tabular}{|c|c|c|c|c|c|c|c|c|c|c|c|c|}
\hline \multirow[b]{3}{*}{ Group } & \multicolumn{6}{|c|}{ No MRF Stimulation } & \multicolumn{6}{|c|}{ MRF Stimulation } \\
\hline & \multicolumn{3}{|c|}{ Tone } & \multicolumn{3}{|c|}{ No Tone } & \multicolumn{3}{|c|}{ Tone } & \multicolumn{3}{|c|}{ No Tone } \\
\hline & $\mathrm{N}$ & Mean & SE & $\mathrm{N}$ & Mean & SE & $\mathrm{N}$ & Mean & SE & $\mathrm{N}$ & Mean & SE \\
\hline TSFS & 5 & 409.20 & 118.42 & 5 & 453.60 & 100.27 & 6 & 424.50 & 90.47 & 8 & 430.50 & 83.19 \\
\hline TWFS & 8 & 194.25 & 89.99 & 5 & 218.00 & 97.81 & 8 & 156.00 & 46.77 & 6 & 456.67 & 64.99 \\
\hline TSFS/ECS & 5 & 311.20 & 89.77 & 4 & 192.00 & 65.28 & 9 & 334.22 & 67.90 & 5 & 177.20 & 63.61 \\
\hline NCSFS/ECS & 6 & 60.67 & 23.28 & 6 & 125.33 & 55.21 & 7 & 50.86 & 23.55 & 5 & 121.40 & 39.05 \\
\hline
\end{tabular}

Note-Explanation of group designations may be found in the text. 
clusion of the experiment. Suppression ratios (DeVietti $\&$ Porter, 1969) were calculated from the heart-rate data with the formula $2 \mathrm{C} /(2 \mathrm{C}+\mathrm{A}+\mathrm{B}) \times 100$, where $C$ refers to the target 15 -sec interval of heart rate, say, following MRF stimulation, and A and B refer, respectively, to the two 15 -sec periods of heart-rate activity recorded during the last $30 \mathrm{sec}$ of the first minute of the reminder session. This transformation yields a score of 50 if there is no change in heart rate during the target $15 \mathrm{sec}$ relative to the baseline recording, a score greater than 50 if heart rate increases during the target interval, and a score less than 50 if heart rate decreases during the target interval.

Analysis of the suppression ratios obtained in the four groups following stimulation or no stimulation of the MRF in the reminder session with a 2 by 4 unweighted means analysis of variance indicated that animals, overall, showed a small, but reliable, decrease in heart rate following MRF stimulation $[F(1,75)=$ $3.81, p=.05]$. Neither the effect of groups nor the interaction reached statistical reliability.

The analysis of the suppression ratios during the presentation of the tone or no tone in reminder was analyzed with a 2 by 2 by 4 unweighted means analysis of variance, with factors identical to those in the analysis of the drink-latency data. This analysis revealed that, overall, animals decreased heart rate to the presentation of the tone $[F(1,66)=64.48, p<$ .01]. Also, the interaction among groups, MRF stimulation (present, absent), and tone (present, absent) was reliable $[F(3,66)=4.01, p=.01]$. No other source approached statistical reliability. The analysis of the simple main effects of the three-way interaction made it clear that the decrease in heart rate to the MRF stimulation confounded the reactivity to the tone. In light of the effects observed in the analysis of the drink-latency data reported above, we chose to analyze the suppression ratios to the reminder session tone (present, absent) only for animals that did not receive preceding stimulation of the MRF. In this way, we avoided the confounding of the MRF stimulation on reactivity to the tone. Suppression ratios for this analysis were calculated as described above, except that the baseline rate used in the ratio was heart rate recorded during the $15-\mathrm{sec}$ interval immediately preceding the tone or no-tone presentation. This was done to ensure that there were no spurious effects resulting from baseline shift between the last $30 \mathrm{sec}$ of the first minute of the reminder session and the presentation of the tone, or no tone, an interval of $75 \mathrm{sec}$. In fact, this precaution turned out to be unnecessary, as analysis of the suppression ratios calculated with either interval of baseline yielded an identical pattern of results.

The suppression ratios, calculated with the 15 -sec baseline immediately preceding the tone or no tone, are shown in Table 2 , and a 2 by 4 unweighted means analysis revealed effects of groups $[\mathrm{F}(3,30)=3.11$,
Table 2

Heart-Rate Suppression to Tone or No Tone in Reminder (No MRF Stimulation) Session in Experiment 1

\begin{tabular}{lccrrrrr}
\hline & \multicolumn{3}{c}{ Tone } & & \multicolumn{3}{c}{ No Tone } \\
\cline { 2 - 4 } \cline { 6 - 8 } \multicolumn{1}{c}{ Group } & $\mathrm{N}$ & Mean & SE & & N & Mean & SE \\
\hline TSFS & 5 & 44.20 & 1.59 & & 4 & 50.00 & .41 \\
TWFS & 5 & 47.20 & .92 & 5 & 49.80 & .37 \\
TSFS/ECS & 5 & 46.00 & .41 & 4 & 51.00 & .71 \\
NCSFS/ECS & 5 & 49.20 & .80 & & 5 & 50.00 & .32 \\
\hline
\end{tabular}

$\mathrm{p}<.05]$, tone (present, absent) $[\mathrm{F}(1,30)=37.42$, $\mathrm{p}<.01]$, and the interaction of these two variables $[\mathrm{F}(3,30=3.87, \mathrm{p}<.02]$. The analysis of the simple main effects of this interaction indicated that each group, except the NCSFS/ECS group, showed more heart-rate suppression to the tone than the same group not receiving the tone in the reminder session (ps $<.03$ ). Also, as part of the analysis of the interaction, differences among the four groups given the tone in reminder were obtained, but no differences among the four groups not given the tone were found. Specific comparisons among the groups given the tone were evaluated with the Newman-Keuls test and demonstrated that the TSFS animals suppressed more to the tone than either the TWFS or NCSFS/ ECS groups (ps $<.05)$, but did not differ from the TSFS/ECS animals. Also, the TSFS/ECS showed more suppression to the tone than the NCSFS/ECS group $(\mathrm{p}<.05)$. No other differences were statistically reliable.

\section{Discussion}

The results of the drink latencies in the presence of the test tone indicated that the presentation of the tone as reminder served to increase retention in TSFS/ECS animals. Also, heart-rate reactivity to the reminder tone was observed in TSFS/ECS animals, indicating that this measure may be useful in predicting the behavioral test performance of these animals.

It is difficult to argue that the relevant reminder treatment, that is, placement of the animals in the original training apparatus and presentation of the original training tone, served as a learning trial. Operationally, this procedure defines an extinction trial as footshock was not given in the reminder session. Indeed, the test performance of all groups, except the TSFS/ECS group, showed decreased drink latencies following this reminder treatment, the TWFS animals showing a statistically reliable decrease. These results support the idea that the reminder treatment served as an extinction trial in the trained groups and as an adaptation trial in the NCSFS/ECS group. In sharp contrast with the general decrease in test performance in all other groups following the reminder tone, this treatment resulted in a reliable increase in test performance in the TSFS/ECS animals. 
These results are contrary to the prediction of the generalization hypothesis that reminder should have equal effects in animals demonstrating weak retention from weak training or strong training and ECS. The present data, as well as two other reports from our laboratory demonstrating a differential reminder effect in TSFS/ECS and TWFS animals (DeVietti \& Haynes, 1975; DeVietti \& Kirkpatrick, 1978), suggest that reminder increased retention in ECS animals by encouraging the return of a stored, but normally unretrievable, memory rather than by summating with a weak memory trace.

In the present study, the presentation of the tone in reminder was more crucial to the reminder effect than was stimulation of the MRF preceding the tone. Because of the cutoff criterion imposed in the test, it is not clear from these data that MRF stimulation had no effect.

We did not expect to find a strong influence of the reminder tone in TSFS/ECS animals in the present study, as we had tested for this effect in two previous investigations and failed to obtain unequivocal evidence that the reminder tone alone increased test performance in ECS animals (DeVietti \& Hopfer, 1974; DeVietti \& Kirkpatrick, 1978).

In examining the procedures used in the present study and in our previous studies, two major differences became apparent. In the present experiment, the animals were fixed with heart-rate recording electrodes and were adapted to wearing a saddle for the collection of heart-rate data. Also, and more important, we suspect, in the present study the reminder tone was presented $135 \mathrm{sec}$ after the animal was placed into the original training apparatus. In our previous work (DeVietti \& Hopfer, 1974; DeVietti \& Kirkpatrick, 1978), the reminder tone was presented $60 \mathrm{sec}$ after placement in this apparatus. Sara, David-Remacle, and Lefevre (1975) reported that ECS-treated animals that were prevented from responding in a passive avoidance test for $30 \mathrm{sec}$ showed better retention of training than ECS animals tested as usual. They interpreted these results as indicating that ECS spared the memory trace of acquisition but modified memory retrieval systems such that ECS animals required time to integrate the memory, in the presence of relevant training cues, into existing functional systems. This result and interpretation is consistent with an earlier report by Adams and Calhoun (1972).

In light of these reports, it may be that the longer interval to the presentation of the tone in the present experiment, relative to our earlier procedure, encouraged memory retrieval in ECS animals.

\section{EXPERIMENT 2}

Experiment 2 was designed to test the notion that the interval between placement in the original training chamber in reminder treatment and the presentation of the reminder tone is critical to the reminder effect. Heart-rate activity was recorded to determine if autonomic reactivity to the reminder tone was related to latency-to-drink performance in the test and to equate procedures between this experiment and Experiment 1 . For this reason, all animals were implanted with unilateral electrodes aimed at the MRF.

\section{Method}

This experiment was identical to Experiment 1, with the exception of the reminder session. As before, the reminder session lasted $180 \mathrm{sec}$, but in the present experiment reminder consisted of the presentation of the reminder tone $60 \mathrm{sec}$ after placement in the training chamber. The animals not given the tone were placed in the cardboard box for $180 \mathrm{sec}$. Although all animals were fitted with the electrode stimulating cable in the reminder session, no stimulation of the MRF was given to any animal. Heart rate was recorded during the entire 180 -sec session.

\section{Results}

The electrode placements tended to cluster in the MRF, as described in Experiment 1.

Analysis of the drink latencies. Once again, latencies to drink in the presence of the test tone were extremely variable. They are shown in the left half of Table 3 . These data were analyzed with a 2 by 4 unweighted means analysis of variance and indicated an effect of groups $[F(3,80)=5.43, p<.01]$, but neither the tone (present or absent) nor the interaction approached statistical reliability. The Newman-Keuls statistical test indicated that, overall, the drink latencies of the

Table 3

Performance of Groups in Experiment 2

\begin{tabular}{|c|c|c|c|c|c|c|c|c|c|c|c|c|}
\hline \multirow[b]{3}{*}{ Group } & \multicolumn{6}{|c|}{$\begin{array}{c}\text { Drink Latencies in the Presence } \\
\text { of the Test Tone }\end{array}$} & \multicolumn{6}{|c|}{$\begin{array}{l}\text { Heart-Rate Suppression to Tone or } \\
\text { No Tone in Reminder Session }\end{array}$} \\
\hline & \multicolumn{3}{|c|}{ Tone } & \multicolumn{3}{|c|}{ No Tone } & \multicolumn{3}{|c|}{ Tone } & \multicolumn{3}{|c|}{ No Tone } \\
\hline & $\mathbf{N}$ & Mean & SE & $\mathbf{N}$ & Mean & SE & $\mathbf{N}$ & Mean & SE & $\mathbf{N}$ & Mean & SE \\
\hline TSFS & 13 & 395.46 & 65.69 & 9 & 372.55 & 74.15 & 12 & 46.17 & .68 & 8 & 50.37 & .37 \\
\hline TWFS & 16 & 249.00 & 58.29 & 11 & 203.73 & 55.17 & 15 & 46.33 & .54 & 8 & 49.75 & .16 \\
\hline TSFS/ECS & 10 & 207.33 & 62.29 & 9 & 208.89 & 70.33 & 9 & 48.11 & .63 & 8 & 50.37 & .18 \\
\hline NCSFS/ECS & 11 & 88.64 & 30.91 & 9 & 191.78 & 63.33 & 9 & 48.89 & .39 & 8 & 50.75 & .45 \\
\hline
\end{tabular}

Note-No stimulation of the MRF was administered to any group. 
TSFS group were longer than those of the other three groups ( $\mathrm{ps}<.05)$, whereas, although trends are obvious, the other three groups did not differ significantly from one another.

Analysis of heart-rate reactivity. The suppression ratios calculated from the baseline data and the heartrate reactivity to the presentation of the tone or no tone in the reminder session are shown in the right half of Table 3 . These data were evaluated with a 2 by 4 unweighted means analysis of variance and revealed an effect of groups $[\mathrm{F}(3,69)=4.74, \mathrm{p}<.01]$, an effect of tone (present, absent) $[\mathrm{F}(3,69)=58.74$, $\mathrm{p}<.01$, and an interaction between these two variables $[F(3,69)=5.19, p<.01]$. The analysis of the simple main effects of this interaction indicated that each group showed more suppression to the presentation of the tone in the reminder session than the corresponding group, which did not receive the tone (ps < .03). Also, no differences were obtained among the four groups not given the tone in reminder, whereas differences in suppression among the four groups presented with the reminder tone were found. Specific comparisons among these four groups with the Newman-Keuls test indicated that the TSFS group suppressed more to the tone than did either the TSFS/ECS group or the NCSFS/ECS group (ps $<$ .05 ) but did not differ from the TWFS group. In addition, the TWFS group showed more suppression than either the TSFS/ECS group or the NCSFS/ECS group (ps $<.05$ ). No difference was discernible between the TSFS/ECS and NCSFS/ECS groups.

A series of $t$ tests was performed to compare the suppression ratios in response to the tone in the reminder session in the four groups in Experiments 1 and 2. The results of these comparisons confirm the impression gained by viewing Tables 2 and 3, that the heart-rate suppression in the TSFS/ECS animals was reliably greater when the reminder tone was presented $135 \mathrm{sec}$ after placement in the apparatus $[\mathrm{t}(12)=2.35$, $\mathrm{p}<.05$ ] than it was when it was given $60 \mathrm{sec}$ after placement in the apparatus. Similar comparisons among the three other groups failed to yield reliable differences in suppression performance between experiments.

\section{Discussion}

The results of this experiment failed to demonstrate that the presentation of the reminder tone $60 \mathrm{sec}$ after placement in the original training apparatus affected drink latency performance in the presence of the test tone in any of the four groups. These data contrast with the findings of Experiment 1, particularly with reference to TSFS/ECS animals. Moreover, the analysis of the heart-rate reactivity of the four groups to the reminder tone yielded a pattern of results quite different from that obtained in Experiment 1. In the present experiment, TSFS/ECS animals showed little, if any, suppression of heart rate to the presentation of the reminder tone that could reasonably be attributed to memory of previous training rather than to startle, and this experience did not lead to improved retention in the behavioral test. Thus, once again, the autonomic reactivity to the reminder tone predicted the behavioral test performance in the TSFS/ECS animals.

The drink latencies of the TSFS and TWFS groups, but not the NCSFS/ECS group, tended to increase, although not significantly, following reminder tone, in contrast with the decrease in test performance in these groups noted in Experiment 1. This may indicate that the shorter interval prior to the presentation of the reminder tone in the present experiment modified the impact of this procedure as an effective extinction trial in trained animals not given in ECS.

\section{GENERAL DISCUSSION}

Heart rates and drink latencies of TSFS/ECS animals given the reminder tone were quite different in Experiments 1 and 2. It is not clear from these experiments whether presentation of the tone was necessary for these effects, as tone presentation was confounded with the training environment in both experiments. However, the heart-rate data strongly suggest that reactivity to the reminder tone was a critical aspect of the effective reminder treatment, as no changes in heart rate were noted in intervals recorded prior to the presentation of the tone. Moreover, it is not entirely clear as to which interval, that is, the interval preceding or that following the reminder tone, was critical to the differential performance of TSFS/ECS animals in the two experiments. Although the duration of the reminder session was a constant between experiments, both the interval to tone onset and the interval following tone offset varied systematically between experiments. Thus, it is possible that either of these two intervals, or some interaction of them, effected the differential results in the TSFS/ ECS animals in the two experiments. We favor the notion that the interval to the onset of the reminder tone is the more critical factor on at least two grounds. First, the heart-rate reactivity to the tone clearly depended on this interval, and second, heart-rate recordings following tone offset indicated that TSFS/ ECS animals in Experiment 1 maintained suppression for at least $30 \mathrm{sec}$, whereas animals in Experiment 2 never did show suppression during the entire $105 \mathrm{sec}$ following tone offset.

Further work will be necessary to determine the effectiveness of the training environment alone as a reminder treatment and to establish that the interval preceding the reminder tone is the critical variable controlling the differential results obtained in TSFS/ ECS animals in Experiments 1 and 2. However, the 
facts remain that, when the interval to reminder tone onset was short (60 sec, Experiment 2), no heart-rate reactivity was noted in TSFS/ECS animals and no evidence of a reminder effect was obtained in these animals in the behavioral test the following day. On the other hand, when the interval to reminder tone onset was long (135 sec, Experiment 1), heart-rate reactivity to the tone was observed and increased drink latencies were found in the behavioral test.

Both the heart-rate reactivity and the drink-latency data strongly suggest that the interval preceding, and possibly that following the nominal reminder stimulus plays a crucial role in determining the effects of this stimulus in TSFS/ECS animals. Furthermore, these experiments suggest that heart-rate reactivity obtained in the reminder session may serve as a useful index in predicting the behavioral performance of ECS animals in the retention test. This relationship does not appear to hold for the other groups in these experiments when only the heart-rate reactivity to the tone is considered. For example, in Experiment 1, TSFS/ECS and TWFS animals showed similar suppression of heart rate to the reminder tone. Yet, the performance of one of these two groups was the reverse of that of the other group, with weakly trained animals showing decreased drink latencies and ECS animals showing increased drink latencies. In short, the same degree of heart-rate suppression to the reminder tone in these groups was followed by very different retention performances. However, the pattern of heart-rate reactivity recorded during the two 15 -sec intervals following the reminder tone differed markedly in these two groups. ECS animals continued to show significant heart-rate suppression in these recorded intervals, whereas weakly trained animals, as well as strongly trained animals not given ECS, showed recovery of heart-rate suppression in the second interval. Moreover, there was a difference in the recovery function between the weakly trained and the strongly trained animals, with the weakly trained animals returning to baseline in the first interval following the tone and remaining at that level during the second interval. In contrast, strongly trained animals still showed significant heart-rate suppression in the first interval following the tone but recovery of suppression in the second interval. Clearly, therefore, while heart-rate suppression during the reminder tone did not differentiate well among the three trained groups, the pattern of recovery of heart rate following the tone did. The different recovery functions suggest that the reminder tone had a different impact in the three groups. It may be that the pattern of heart-rate recovery in the weakly and strongly trained animals reflected retention of weak and strong training, respectively. On the other hand, the sustained heart-rate suppression in the case of ECS animals may indicate at least a partial recollection of strong training and perhaps is indicative of further memory processing in this group.
We submit that the results of the present experiments strongly favor the idea that ECS modifies memory retrieval and that a careful analysis of the conditions sufficient to facilitate retention in animals rendered amnesic by posttraining treatments may increase our understanding of memory processes.

\section{REFERENCES}

Adams, H. E., \& Calhoun, K. S. Indices of memory recovery following electroconvulsive shock. Physiology \& Behavior, 1972, 9, 783-787.

Cherkin, A. Retrograde amnesia in the chick: Resistance to the reminder effect. Physiology \& Behavior, 1972, 8, 949-955.

DeVietti, T. L., \& Haynes, D. Reminder: Similar and differential effects in amnesic and weakly trained rats. Physiological Psychology, 1975, 3, 265-269.

DeVietti, T. L., \& Hopfer, T. M. Complete amnesia induced by ECS and complete recovery of memory following reinstatement treatment. Physiology \& Behavior, 1974, 12, 599-603.

DeVietti, T. L., \& Kirkpatrick, B. R. Reminder induced by training cues and stimulation of the mesencephalic reticular formation. Physiological Psychology, 1978, 3, 381-386.

DeVietti, T. L., \& Larson, R. C. ECS effects: Evidence supporting state-dependent learning in rats. Journal of Comparative and Physiological Psychology, 1971, 74, 407-415.

DeVietti, T. L., \& Porter, P. B. Modification of the autonomic component of the conditioned emotional response. Psychological Reports, 1969, 24, 951-958.

Gold, P. E., Haycock, J. W., Macri, J., \& McGaugh, J. L. Retrograde amnesia and the "reminder effect": An alternative interpretation. Science, 1973, 180, 1199-1201.

Gold, P. E., \& KING, R. A. Retrograde amnesia: Storage failure versus retrieval failure. Psychological Bulletin, 1974, 81, 465-469.

HAyсоск, J. W., Gold, P. E., MACri, J., \& McGaUGH, J. L. Noncontingent footshock attenuation of retrograde amnesia: A generalization effect. Physiology \& Behavior, 1973, 11, 99-101.

HAYES, K. J. Cognitive and emotional effects of electroconvulsive shock in rats. Journal of Comparative and Physiological Psychology, 1948, 41, 40-61.

Kesner, R. P., \& Conner, H. S. Cue-dependent recovery from ECS-induced amnesia: Evidence for time dependence. Physiological Psychology, 1974, 2, 123-125.

KIRK, R. E. Experimental design: Procedures for the behavioral sciences. Belmont, Calif: Wadsworth, 1968.

LEwis, D. J. Sources of experimental amnesia. Psychological Review, 1969, 76, 461-472.

LEWIS, D. J. A cognitive approach to experimental amnesia. American Journal of Psychology, 1976, 89, 51-80.

Miller, R. R., \& Springer, A. D. Amnesia, consolidation, and retrieval. Psychological Review, 1973, 80, 69-79.

Miller, R. R., \& SPringer, A. D. Implications of recovery from experimental amnesia. Psychological Review, 1974, 81, 470-473.

Pellegrino, L. J., \& Cushman, A. J. A stereotaxic atlas of the rat brain. New York: Appleton-Century-Crofts, 1967.

Quartermain, D., McEwen, B. S., \& Azmitia, E. C. Recovery of memory following amnesia in the rat and mouse. Journal of Comparative and Physiological Psychology, 1972, 79, 360-370.

Sara, S. J., David-Remacle, M., \& Lefevre, D. Passive avoidance behavior in rats after electroconvulsive shock: Facilitative effect of response retardation. Journal of Comparative and Physiological Psychology, 1975, 89, 489-497.

Thomson, J. H., Beeman, F. J., Conger, G. L., Reeder, W. T., Steinhilb, K. R., Wittman, T. K., \& DeVietti, T. L. Videotape technology in the identification and storage of intracerebral electrode locations. Physiology \& Behavior, 1978, 20, 487-489.

(Received for publication March 17, 1980; revision accepted July 29, 1980.) 